\title{
Phase transitions for laminar-turbulent flows in a pipeline or through porous media
}

\author{
Andrea Corli and Haitao Fan
}

Dedicated to Professor Marshall Slemrod on occasion of his 70th birthday

\begin{abstract}
Liquid/vapor phase changes for a fluid flow through a porous medium or a pipeline are considered. In particular, the model covers both laminar and turbulent flows. The presence of both laminar and turbulent flows causes jump discontinuities in the friction coefficient. Classical trajectories of traveling waves terminate when they intersect the discontinuity. We construct traveling wave solutions by monotonically smoothing the discontinuity and then taking a limiting process. The limit is independent of the monotonepreserving smoothing. This uniqueness justifies the construction of the traveling wave via this smoothing and limiting approach. Existence of traveling waves is established in a wide range of situations; in particular, the end states may be formed either by pure phases or mixtures.
\end{abstract}

\section{Introduction}

In this paper we consider the isothermal inviscid fluid flow through a porous medium or a pipeline, in presence of liquid-vapor phase changes. A system of evolution equations governing such flows in Lagrangian coordinates is

$$
\left\{\begin{array}{l}
v_{t}-u_{x}=0, \\
u_{t}+p(v, \lambda)_{x}=-\alpha(u) u, \\
\lambda_{t}=\frac{1}{\tau}\left(p(v, \lambda)-p_{e}\right) \lambda(\lambda-1),
\end{array}\right.
$$

for $t>0$ and $x \in \mathbb{R}$. Here, $v>0$ denotes the specific volume, $u$ the velocity, $p$ the pressure, $\lambda \in[0,1]$ the mass-density fraction of the vapor in the fluid. The constants $\tau>0$ and $p_{e}>0$ are the characteristic reaction time and the equilibrium pressure, respectively.

The pressure function $p(v, \lambda)>0$ is assumed to be of class $C^{2}$ and satisfies

$$
p_{v}<0, \quad p_{\lambda}>0, \quad p_{v v}>0, \quad p_{v \lambda}<0 .
$$


Physical pressure functions satisfy, in addition to (1.2), also the conditions

$$
\lim _{v \rightarrow 0+} p(v, \lambda)=+\infty, \quad \lim _{v \rightarrow+\infty} p(v, \lambda)=0,
$$

for every $\lambda \in[0,1]$. A simple example of a pressure law satisfying both (1.2) and (1.3) is, for $\gamma \geq 1$,

$$
p=\frac{1+\lambda}{v^{\gamma}} .
$$

The function $\alpha(u)>0$ is the friction coefficient exerted onto the fluid by the porous media or the pipe; to discuss this coefficient let us focus on the case of a flow through a pipeline [44, 45], the case of a flow in porous media being analogous. We completely disregard any discussion on the diameter, length and roughness of the pipeline, which are important parameters but are here considered to be fixed. When the flow speed is slow, then the flow is laminar; however, the flow becomes turbulent for higher speeds. The friction coefficient for a turbulent flow, which is usually computed by using the Colebrook interpolation formula [45, (6.64)], is larger than that of a laminar flow; both are represented in what is called a Moody chart [45, Fig. 6.13]. The main point is that the friction coefficient suffers a discontinuity when passing from a laminar regime to a turbulent regime. Indeed, there is a narrow zone between the two regimes where there is no reliable value for the friction factor [45, page 348]; in our model we simply admit that this transition zone is reduced to a single point. Therefore, we can model the "Moody" friction coefficient $\alpha(u)$ as follows:

$$
\alpha(u)= \begin{cases}a & \text { if }|u|<u^{*}, \\ b|u| & \text { if }|u| \geq u^{*},\end{cases}
$$

for some $a>0, b>0, u^{*}>0$ and $a<b u^{*}[32,33,44,45]$. One result of this paper is that the details of $\alpha(u)$ in the narrow transition zone do not matter much.

We now briefly discuss the papers related to system (1.1). When there are no phase transitions in the flow, then the third equation is missing and $p$ only depends on $v$. The corresponding $2 \times 2$ system has been studied by many authors: see $[36,13,28,29,37,14,30]$, for the homogeneous case or with continuous source terms and $[32,33]$ for the case of discontinuous source terms.

Here, we consider phase change flows in a pipe or porous media in both laminar and turbulent regions. One approach is to study the hyperbolicelliptic mixed type $p$-system with the damping term $-\alpha u$. The study of 
hyperbolic-elliptic mixed type $p$-system was pioneered by Slemrod [41, 42, 43], Shearer [39, 40] and followed later by many authors. For more references, please see [20]. We are not aware of any attempt done in this approach. This is an interesting direction for future study.

Another approach to consider phase changes is to study the full $3 \times 3$ system (1.1). We refer to $[1,5]$ for the homogeneous case; see also [3] for a large data analysis. About the special case $\alpha \equiv 0$, the Riemann problem was solved in [8] if $\tau=0$ while the relaxation limit $\tau \rightarrow 0$ was investigated in [2]; moreover, all possible traveling waves were characterized in [16]. When a diffusion term $\lambda_{x x}$ is added to the right side of the third equation in (1.1), traveling waves were obtained in $[15,18,19]$. Using these traveling waves, the system was found to have solutions exhibiting phenomena observed in actual experiments [17].

The case when $\alpha$ is a positive constant was considered in [11], where traveling waves of system (1.1) were obtained for a large range of end states. Entirely analogous results are valid if $\alpha$ depends on $\lambda$ but is bounded away from zero [9]. Moreover, in [9] we proved that, under such an assumption, system (1.1) satisfies the Shizuta-Kawashima condition and is strictly entropy-dissipative; as a consequence [24], the initial-value problem has smooth global solutions if the initial data are close either to the stableliquid phase $\left(\lambda=0\right.$ and $\left.p>p_{e}\right)$ or to the stable-vapor phase $(\lambda=1$ and $\left.p<p_{e}\right)$. In [10], we continued the study of system (1.1) in the case $\alpha$ depends on $\lambda$ in the degenerate situation

$$
\alpha(\lambda)>0, \quad \text { for } \lambda \in[0,1), \quad \alpha(1)=0 .
$$

The condition $\alpha(1)=0$ assumes that the porous medium exerts no friction on vapor flows. We obtained various traveling waves for (1.1), many of them were not present when $\alpha$ is bounded away from 0 .

The friction coefficient $\alpha$ can also depend on $u$. For example, when the flow speed is slow, the flow is laminar and the friction coefficient is small and almost constant. In the model proposed above, as the speed of the fluid flow crosses a threshold, the flow becomes turbulent and the friction coefficient increases sharply across the threshold. For system (1.1) with discontinuous friction coefficient but without phase changes, Luskin [32] proved the global existence of smooth solutions with initial data in an invariant region and whose first derivative is small enough. Subsequently, Luskin and Temple [33] studied the existence of global weak solutions.

In this paper, we investigate traveling waves of (1.1) with a discontinuous friction coefficient $\alpha(u)$ as in (1.4). Then, we are led to consider a 
dynamical system with a discontinuous right-hand side; we refer to [21] for a thorough account on this subject. Now, we briefly account about traveling waves that solve dynamical systems with discontinuous right-hand side. A famous example occurs in combustion models, see [7] and references quoted there. In particular, in that paper the existence of solutions is proved either by a shooting argument (in the scalar case) or by energy estimates (in the case of a $2 \times 2$ system). That paper gave rise to a wide area of research that cannot be accounted here. However, we quote [22] for recent mathematical results on combustion phenomena in porous media. For system of balance laws, sources that are discontinuous in the state variables have been considered, to the best of our knowledge, only in the resonant case, see $[31,25,26,4,23]$.

With respect to our earlier papers $[11,10]$, the difficulty is that the trajectories of the traveling waves of (1.1) terminate when they meet the discontinuity of $\alpha(u)$. So, we have to define traveling waves of (1.1) in a new, reasonable way to extend them and complete the connections between equilibrium points. This is done as follows.

In our model, the discontinuity of $\alpha(u)$ is a simplification that models the sharp increase of $\alpha$ when the flow speed increases from the laminar region to the turbulent region. Indeed, as we mentioned above, in real phenomena the sharp increase occurs for speeds in a narrow interval. A physically meaningful way to resolve the terminations of the traveling wave trajectories consists in smoothly connecting the discontinuity of $\alpha(u)$ over a narrow interval of width $\epsilon>0$ and then let $\epsilon \rightarrow 0+$. This can be understood as the first step of the approach. The second step is to establish the existence of traveling waves, denoted as $\left(v_{\epsilon}, \lambda_{\epsilon}\right)(\xi)$, to (1.1) with the above smoothed $\alpha$. At last, in the third step one must show the existence of the $\operatorname{limit}_{\epsilon \rightarrow 0+}\left(v_{\epsilon}, \lambda_{\epsilon}\right)(\xi)=:(v, \lambda)(\xi)$ and prove that it is independent of the monotone smoothing of $\alpha(u)$. Here, a smoothing of $\alpha(u)$ is called monotone if it is increasing (or decreasing) in the smoothing region where $\alpha\left(u^{*}-\right)<\alpha\left(u^{*}+\right)$ (or $\alpha\left(u^{*}-\right)>\alpha\left(u^{*}+\right)$, respectively). This includes a wide range of smoothings for $\alpha(u)$. This independence essentially overcomes the problem that, as we wrote above, there is no reliable model for $\alpha$ in the transition zone. Our estimate shows that solutions corresponding to two different smoothings of width $\epsilon>0$ differ by at most $O(1) \epsilon$. Moreover, one must prove that the limit $(v, \lambda)(\xi)$ satisfies the dynamical system corresponding to the traveling wave profiles of (1.1) in the sense of the differential inclusions discussed, for instance, in [21]. We call solutions in the sense of vanishing smoothing the traveling wave solutions of (1.1) constructed in this way. The aim of this paper is to carry out the 
vanishing smoothing approach to establish the existence of traveling waves to (1.1), for a wide range of end states.

In Section 2 we formulate the problem and state the main results. A short Section 3 follows, where we collect some preliminary results. The core of the paper is Section 4, where we provide fully detailed proofs for one of the possible five cases that can occur; in this section we also discuss the overall structure of the vanishing smoothing solutions. The final Section 5 contains sketches of the proofs for the remaining cases.

\section{Main results}

A traveling wave to (1.1) with constant speed $c$ is a solution to (1.1) of the form

$$
U(\xi)=U\left(\frac{x-c t}{\tau}\right),
$$

whose end-states at $\pm \infty$ are imposed together with the vanishing of the first derivatives. As a consequence $U$ must solve

$$
\left\{\begin{array}{l}
-c v^{\prime}-u^{\prime}=0, \\
-c u^{\prime}+p^{\prime}=-A(u) u, \\
-c \lambda^{\prime}=\left(p-p_{e}\right) \lambda(\lambda-1),
\end{array}\right.
$$

together with

$$
\left\{\begin{array}{l}
(v, u, \lambda)( \pm \infty)=\left(v_{ \pm}, u_{ \pm}, \lambda_{ \pm}\right), \\
\left(v^{\prime}, u^{\prime}, \lambda^{\prime}\right)( \pm \infty)=0
\end{array}\right.
$$

for $\left(v_{ \pm}, u_{ \pm}, \lambda_{ \pm}\right) \in(0, \infty) \times(-\infty, \infty) \times[0,1]$. Here above, "' " denotes differentiation with respect to $\xi, p^{\prime}=p_{v} v^{\prime}+p_{\lambda} \lambda^{\prime}$ and

$$
A(u)=\alpha(u) \tau .
$$

In the case $A=0$, the study of the traveling wave system (2.1) was done in [15]. Here, we investigate the case $A=A(u)>0$.

If a solution to (2.1)-(2.2) exists, we say that $U_{-}=\left(v_{-}, u_{-}, \lambda_{-}\right) \rightarrow$ $\left(v_{+}, u_{+}, \lambda_{+}\right)=U_{+}$is a connection with speed $c$. We focus our analysis on the case $c \geq 0$; indeed, if $U$ is a connection $U_{-} \rightarrow U_{+}$with speed $c$, then $\tilde{U}(\xi) \doteq(v(-\xi),-u(-\xi), \lambda(-\xi))$ is a connection $\tilde{U}_{-}=\left(v_{+},-u_{+}, \lambda_{+}\right) \rightarrow$ $\tilde{U}_{+}=\left(v_{-},-u_{-}, \lambda_{-}\right)$with speed $-c$. 


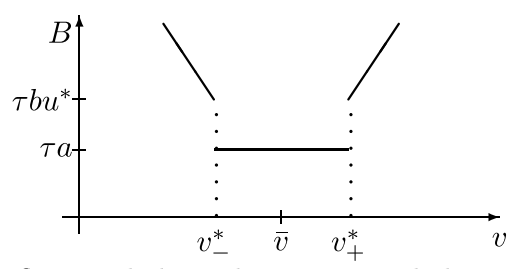

flow: turbulent laminar turbulent

Figure 2.1: Graph of the function $B$.

The end states $\left(v_{ \pm}, u_{ \pm}, \lambda_{ \pm}\right)$in (2.2), being equilibrium points of (2.1), must satisfy

$$
u_{ \pm}=0 \quad \text { and } \quad\left(p_{ \pm}-p_{e}\right) \lambda_{ \pm}\left(\lambda_{ \pm}-1\right)=0
$$

The jump condition

$$
c\left(v_{+}-v_{-}\right)=0
$$

which follows from $(2.1)_{1}$ and $(2.3)$, is also necessary for the existence of traveling waves. This leads either to $c=0$ or $v_{-}=v_{+}$. When $c=0$, system (2.1) has only trivial solutions, see Lemmas 2.1 and 5.3 of [11]. Therefore, in the rest of this paper, we consider the case

$$
c>0
$$

and hence, by (2.4),

$$
v_{-}=v_{+}=: \bar{v}
$$

By $(2.1)_{1}$ it follows that $u=-c(v-\bar{v})$ so that $A(u)=A(-c(v-\bar{v}))=: B(v)$. If we denote

$$
v_{ \pm}^{*}:=\bar{v} \pm \frac{u^{*}}{c}
$$

then

$$
B(v)= \begin{cases}\tau a & \text { if } v \in\left(v_{-}^{*}, v_{+}^{*}\right) \\ \tau b c|v-\bar{v}| & \text { if } v \in\left(0, v_{-}^{*}\right] \cup\left[v_{+}^{*}, \infty\right)\end{cases}
$$


Notice that the interval $\left(0, v_{-}^{*}\right]$ may be empty (if $\left.v_{-}^{*} \leq 0\right)$. As a consequence, we can rewrite the traveling wave system (2.1)-(2.2) as

$$
\left\{\begin{array}{l}
\left(c^{2}+p_{v}\right) v^{\prime}=B c(v-\bar{v})+\frac{1}{c} p_{\lambda}\left(p-p_{e}\right) \lambda(\lambda-1), \\
\lambda^{\prime}=-\frac{1}{c}\left(p-p_{e}\right) \lambda(\lambda-1), \\
(v, \lambda)( \pm \infty)=\left(\bar{v}, \lambda_{ \pm}\right)
\end{array}\right.
$$

for $(\lambda, v) \in \Omega:=[0,1] \times(0, \infty)$. We also denote, see Figure 2.3,

$$
\begin{aligned}
& \Omega_{\text {lam }}=\left\{(\lambda, v) \in \Omega: v \in\left(v_{-}^{*}, v_{+}^{*}\right)\right\}, \\
& \Omega_{\text {turb }}=\left\{(\lambda, v) \in \Omega: v \in\left(0, v_{-}^{*}\right] \cup\left[v_{+}^{*}, \infty\right)\right\} .
\end{aligned}
$$

We define

$$
\begin{aligned}
& s(\lambda, v):=c^{2}+p_{v}, \\
& g(\lambda, v):=B c^{2}(v-\bar{v})+p_{\lambda}\left(p-p_{e}\right) \lambda(\lambda-1),
\end{aligned}
$$

so that system (2.7) can be written in $\Omega$ as

$$
\left\{\begin{array}{l}
c s v^{\prime}=g, \\
\lambda^{\prime}=-\frac{1}{c}\left(p-p_{e}\right) \lambda(\lambda-1), \\
(v, \lambda)( \pm \infty)=\left(\bar{v}, \lambda_{ \pm}\right) .
\end{array}\right.
$$

Lemma 2.1. Assume $A>0$ and $c>0$. For a traveling wave of (2.8) to exist, the end states $\left(\bar{v}, \lambda_{ \pm}\right)$must satisfy one of the following necessary conditions:

(i) $p_{ \pm} \geq p_{e}$ and $\lambda_{-}=0, \lambda_{+}=1$;

(ii) $p_{ \pm} \leq p_{e}$ and $\lambda_{-}=1, \lambda_{+}=0$;

(iii) $p_{+}>p_{e}=p_{-}$and $\lambda_{-}<1, \lambda_{+}=1$;

(iv) $p_{+}<p_{e}=p_{-}$and $\lambda_{-}>0, \lambda_{+}=0$;

(v) $p_{-}=p_{+}=p_{e}$ and $\lambda_{-}=\lambda_{+}$.

The proof is analogous to that given in [11] for an analogous result and then omitted. The end states $\left(\bar{v}, \lambda_{ \pm}\right)$corresponding to cases (i)-(iv) in the above theorem are depicted in Figure 2.2 together with a schematic representations of the connections.

In [11], for constant $A$, traveling waves with end states described in (i)$(v)$ of the above lemma are proved to exist under some conditions involving 


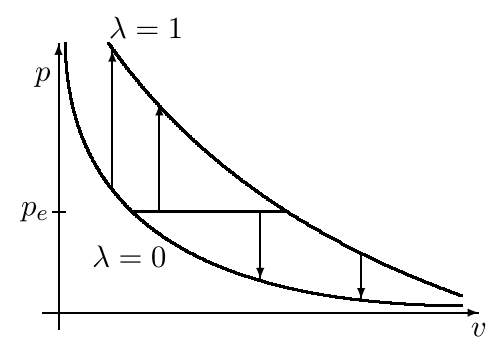

Figure 2.2: Possible end states of traveling wave with $c>0$. Vectors point from $\left(\bar{v}, \lambda_{-}\right)$to $\left(\bar{v}, \lambda_{+}\right)$. After the wave passes the fluid, the fluid state changes from $\left(\bar{v}, \lambda_{+}\right)$to $\left(\bar{v}, \lambda_{-}\right)$.

the curves

$$
\mathcal{S}=\{(\lambda, v) \in \Omega: s(\lambda, v)=0\} \quad \text { and } \quad \mathcal{P}=\left\{(\lambda, v) \in \Omega: p(v, \lambda)=p_{e}\right\}
$$

In this paper the friction parameter $A=A(u)$ is discontinuous at $u= \pm u^{*}$ and of course is not a constant. Then, the function

$$
g(\lambda, v)=B c^{2}(v-\bar{v})+p_{\lambda}\left(p-p_{e}\right) \lambda(\lambda-1)
$$

is discontinuous, in the plane $(\lambda, v)$, along the lines $v=v_{ \pm}^{*}$ with $v_{ \pm}^{*}>0$. Consider the region

$$
G:=\{(\lambda, v) \in \Omega: g(\lambda, v)>0\}
$$

The boundary $\mathcal{G}$ of $G$ consists of the null set $\mathcal{G}_{0}=\{(\lambda, v) \in \Omega: g(\lambda, v)=0\}$ of $g$ and of the set

$$
\mathcal{G}_{\text {disc }}=\left\{(\lambda, v) \in \Omega: v=v_{ \pm}^{*}, g(\lambda, v+) \cdot g(\lambda, v-) \leq 0\right\}
$$

which corresponds to the jump discontinuities of $g$ across which $g$ changes sign. Therefore

$$
\mathcal{G}=\partial G=\mathcal{G}_{0} \cup \mathcal{G}_{\text {disc }}
$$

Classical trajectories of (2.8) terminate when they intersect the lines $v=v_{ \pm}^{*}$ at points where $s \neq 0$ : differentiability is lost. To overcome this difficulty we extend the definition of traveling wave beyond the classical sense by the following vanishing smoothing approach.

The first step of this approach is smoothing the discontinuities of $A(u)$ around the points of discontinuity $u= \pm u^{*}$, by convolution for example, or 


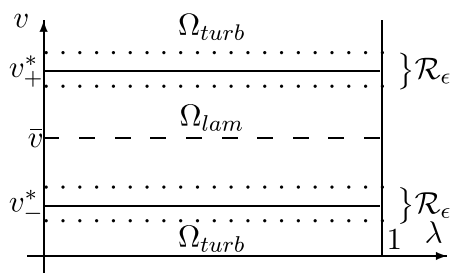

Figure 2.3: Possible discontinuity lines of the function $g$ and the smoothing region $\mathcal{R}_{\epsilon}$. Notice that $v_{-}^{*}$ is missing if it is negative.

equivalently those of $g(\lambda, v)$. Recall that $B$ is discontinuous at $v_{ \pm}^{*}$; then, we define the smoothing region as

$$
\mathcal{R}_{\epsilon}=\left\{(\lambda, v) \in \Omega:\left|v-v_{ \pm}^{*}\right|<\epsilon\right\}
$$

for a small number $\epsilon>0$, see Figure 2.3. We denote the smoothed functions of $B(v)$ and $g(\lambda, v)$ by $B_{\epsilon}(v)$ and

$$
g_{\epsilon}(\lambda, v)=B_{\epsilon} c^{2}(v-\bar{v})+p_{\lambda}\left(p-p_{e}\right) \lambda(\lambda-1),
$$

respectively. In other words, we have $g_{\epsilon}(\lambda, v)=g(\lambda, v)$ outside $\mathcal{R}_{\epsilon}$. We always require that the smoothing is monotone preserving in the sense that

$$
\begin{aligned}
B_{\epsilon}(v) \text { is increasing (decreasing) where } B(v) \text { is increasing } \\
\text { (resp., decreasing). }
\end{aligned}
$$

The second step is establishing the existence of solutions $\left(v_{\epsilon}, \lambda_{\epsilon}\right)(\xi)$ to the problem

$$
\left\{\begin{array}{l}
c s v^{\prime}=g_{\epsilon} \\
\lambda^{\prime}=-\frac{1}{c}\left(p-p_{e}\right) \lambda(\lambda-1) \\
(v, \lambda)( \pm \infty)=\left(\bar{v}, \lambda_{ \pm}\right)
\end{array}\right.
$$

which is deduced by (2.8) by replacing $g$ with $g_{\epsilon}$.

In the third step we shall prove that $\left(v_{\epsilon}, \lambda_{\epsilon}\right)(\xi) \rightarrow(v, \lambda)(\xi)$ a.e. uniformly in $\epsilon$. The limit satisfies (2.8) away from $v=v_{ \pm}^{*}$. At $v=v_{ \pm}^{*}$, the limit satisfies (2.8) in the sense of differential inclusions, see Theorem 2.2 below for the precise statement. The limit is unique in the sense that the limit $(v, \lambda)(\xi)$ is independent of the monotone smoothing. 
The main result of this paper is stated below and roughly runs as follows: In correspondence of each necessary condition in Lemma 2.1 we assume some further sufficient conditions which allow us to prove the existence and uniqueness of traveling waves to (2.8). All the cases in Lemma 2.1 are taken into consideration.

Theorem 2.2. Assume (1.2), (1.4), (2.5), (2.11) and consider end states as in (2.2) with $u_{ \pm}=0$. If one of the following conditions is satisfied, then there is a unique, up to a shift in $\xi$, traveling-wave solution of (1.1) in the sense of the vanishing smoothing. Furthermore, it satisfies (1.1) in the sense of differential inclusion.

(i) $p_{ \pm} \geq p_{e}, \lambda_{-}=0, \lambda_{+}=1$; moreover $\mathcal{G}$ and $\mathcal{S}$ intersect at most once with a transverse intersection.

(ii) $p_{ \pm} \leq p_{e}, \lambda_{-}=1, \lambda_{+}=0$; moreover, $\mathcal{G} \cap \mathcal{S}=\emptyset$ and $B c^{2}+\left(p_{\lambda}\left(p-p_{e}\right)\right)_{v} \times$ $\lambda(\lambda-1)>0$ for $v$ near $v_{-}^{*}$.

(iii) $p_{+}>p_{e}=p_{-}, \lambda_{-}<1, \lambda_{+}=1$; moreover $\mathcal{G}$ and $\mathcal{S}$ intersect at most once in the region $\left(\lambda_{-}, 1\right] \times \mathbb{R}$ with a transverse intersection.

(iv) $p_{+}<p_{e}=p_{-}, \lambda_{-}>0, \lambda_{+}=0$ and $\mathcal{G} \cap \mathcal{S}=\emptyset$.

(v) $p_{-}=p_{+}=p_{e}$ and $\lambda_{-}=\lambda_{+}$. In this case the solution is the trivial constant solution $(v, \lambda)(\xi) \equiv\left(\bar{v}, \lambda_{-}\right)$.

Corollary 2.3. In addition to (1.2), (1.4), (2.5) and (2.11), further assume that the physical assumptions (1.3) hold; moreover, let the end states in (2.2) satisfy $u_{ \pm}=0$ and one of the necessary conditions listed in Lemma 2.1.

Then, if $c>0$ is either sufficiently large or sufficiently small there is a unique (up to a shift in $\xi$ ) traveling-wave solution to (1.1).

\section{Some preliminary results}

We first give a result on the eigenvalues of the equilibrium points of (2.1); its proof is analogous to that given in [11].

Lemma 3.1. Assume (2.5)-(2.6), $s \neq 0$ and consider the linearized system of (2.8) at the equilibrium points; then we have the following.

(i) At the equilibrium point $(\bar{v}, 0)$, the eigenvalues are $\mu_{1}=\frac{B c}{s}$ and $\mu_{2}=$ $\frac{p-p_{e}}{c}$; the corresponding eigenvectors in $(V, \Lambda)$ coordinates are

$$
(1,0) \quad \text { and } \quad\left(\frac{p_{\lambda}\left(p-p_{e}\right)}{c s}, \frac{B c}{s}-\frac{p-p_{e}}{c}\right) \text {. }
$$



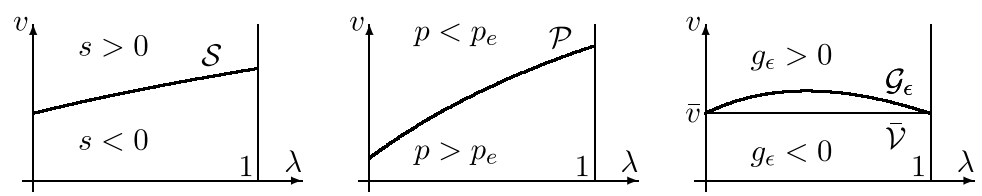

Figure 3.1: Signs of $s, p-p_{e}$, and $g_{\epsilon}$ in the plane $(\lambda, v)$.

(ii) At the equilibrium point $(\bar{v}, 1)$, the eigenvalues are $\mu_{1}=\frac{B c}{s}$ and $\mu_{2}=$ $-\frac{p-p_{e}}{c}$; their corresponding eigenvectors are

$$
(1,0) \quad \text { and } \quad\left(-\frac{p_{\lambda}\left(p-p_{e}\right)}{c s}, \frac{B c}{s}+\frac{p-p_{e}}{c}\right) \text {. }
$$

(iii) Suppose that $(\bar{v}, \bar{\lambda})$ is an equilibrium point of (2.8) with $\bar{\lambda} \neq 0,1$ and $p(\bar{v}, \bar{\lambda})=p_{e} ;$ assume that $\bar{s}:=s(\bar{v}, \bar{\lambda}) \neq 0$. Then, denoting again the eigenvalues by $\mu_{1}$ and $\mu_{2}$, we have that $\mu_{1}<0<\mu_{2}$ if $\bar{s}<0$ and $0<\mu_{2}<\mu_{1}$ if $\bar{s}>0$.

A simple calculation on the equations defining $\mathcal{S}$ and $\mathcal{P}$ shows that these sets are graphs of two smooth functions $v^{\mathcal{S}}(\lambda)$ and $v^{\mathcal{P}}(\lambda)$, respectively. Indeed, we have

$$
\frac{d v^{\mathcal{S}}}{d \lambda}=-\frac{p_{v \lambda}}{p_{v v}}>0 \quad \text { and } \quad \frac{d v^{\mathcal{P}}}{d \lambda}=-\frac{p_{\lambda}}{p_{v}}>0 .
$$

By (2.9) and (2.10) we compute

$$
\begin{aligned}
g_{v} & =B c^{2}+B_{v} c^{2}(v-\bar{v})+\left[p_{\lambda v}\left(p-p_{e}\right)+p_{\lambda} p_{v}\right] \lambda(\lambda-1), \quad v \neq v_{ \pm}^{*} \\
g_{\epsilon, v} & =B_{\epsilon} c^{2}+B_{\epsilon, v} c^{2}(v-\bar{v})+\left[p_{\lambda v}\left(p-p_{e}\right)+p_{\lambda} p_{v}\right] \lambda(\lambda-1)
\end{aligned}
$$

We denote by $\mathcal{G}_{\epsilon}$ the null set $\left\{(\lambda, v) \in \Omega: g_{\epsilon}(\lambda, v)=0\right\}$ of $g_{\epsilon}$. We refer to Figure 3.1 for the signs of the functions $s, p-p_{e}$ and $g_{\epsilon}$. We also denote by $\overline{\mathcal{V}}$ the line $v=\bar{v}$.

About the set $\mathcal{G}$, we notice that $g(0, v)=0$ or $g(1, v)=0$ if and only if $v=\bar{v}$. Moreover, $g(0, v)>0$ if $v>\bar{v}$ and $g(0, v)<0$ if $v<\bar{v}$; the same holds for $g(1, v)$. Since the function $\lambda \mapsto g(\lambda, v)$ is smooth for every $v \in(0, \infty)$, it follows that

$$
\mathcal{G}_{\text {disc }} \subset\left\{(\lambda, v) \in \Omega: \delta<\lambda<1-\delta, v=v_{ \pm}^{*}\right\}
$$

for some $\delta>0$. We also notice that if $\mathcal{G}_{0} \subset[0,1] \times\left(v_{-}^{*}, v_{+}^{*}\right)$, then $\mathcal{G}_{\text {disc }}=\emptyset$. 
The relative positions of the curves $\mathcal{S}, \mathcal{P}, \mathcal{G}_{\epsilon}$ and $\overline{\mathcal{V}}$ are very important in the following. As in [10], we write for short "below" ("above") for "strictly below" (resp., "strictly above"). Moreover, if $\mathcal{A}$ and $\mathcal{B}$ are two sets in the $(\lambda, v)$-plane we write $\mathcal{A} \prec \mathcal{B}(\mathcal{A} \succ \mathcal{B})$ to mean that $\mathcal{A}$ lies below (resp., above) $\mathcal{B}$.

\section{Existence and convergence of solutions $\left(v_{\epsilon}, \lambda_{\epsilon}\right)(\xi)$, Case (i)}

We begin by studying the shape of the boundary $\mathcal{G}$ in the case $\overline{\mathcal{V}} \prec \mathcal{P}$. Denote by $\Omega_{1}$ the region between $\overline{\mathcal{V}}$ and $\mathcal{P}$.

Since $B$, though discontinuous, is increasing in $\Omega_{1}$, by (3.2) we deduce that $v \mapsto g_{v}(\lambda, v)>0$ is increasing in $\Omega_{1}$ for every $\lambda \in[0,1]$. Furthermore, we have $g(\lambda, \bar{v}) \leq 0$ and $g(\lambda, v)>0$ at $\mathcal{P}$. Thus, at points of continuity of $g$ in $\Omega_{1}$, the set $\mathcal{G}_{0}$ is a curve of the form $v=v^{\mathcal{G}}(\lambda)$.

If $\mathcal{P} \prec\left\{v=v_{+}^{*}\right\}$, then also $\mathcal{G}_{0} \prec\left\{v=v_{+}^{*}\right\}$ and $\mathcal{G}_{\text {disc }}=\emptyset$. This case is covered by Theorems 4.1 and 4.3 of [11]: since the direction vectors of the dynamical system (2.8) point downwards at $\mathcal{P}$, every trajectory entirely lies in the laminar-flow regime.

If $\mathcal{P} \nprec\left\{v=v_{+}^{*}\right\}$, then either $\mathcal{P}$ intersects the line $\left\{v=v_{+}^{*}\right\}$ or lies above it. In this case, we claim that the results of [11] still apply if the set $\mathcal{G}_{0}$ entirely lie in $\Omega_{\text {lam }}$. Indeed, from the proof below, we can conclude that the trajectories of (2.8) must lie below $\max \left\{v:(\lambda, v) \in \mathcal{G}_{0}\right\}$ and above $\min \left\{v:(\lambda, v) \in \mathcal{G}_{0}\right\}$, see Figures 4.1 and 4.3. Then, the trajectories will not enter $\Omega_{\text {turb }}$ if $\mathcal{G}_{0}$ entirely lie in $\Omega_{\text {lam }}$ and hence the results of [11] still apply.

Therefore the remaining interesting situation is when both

$$
\mathcal{G}_{0} \cap \Omega_{\text {lam }} \neq \emptyset \quad \text { and } \quad \mathcal{G}_{0} \cap \Omega_{\text {turb }} \neq \emptyset
$$

hold. If (4.1) holds, then $\mathcal{G}_{0} \cap \Omega_{\text {lam }}$ and $\mathcal{G}_{0} \cap \Omega_{\text {turb }}$ are connected by segments lying on the line $\left\{v=v_{+}^{*}\right\}$; these segments form $\mathcal{G}_{\text {disc }}$. Indeed, in any case, we observe that $\mathcal{G}$ and $\mathcal{P}$ can intersect only at $v=\bar{v}$, as well as $\mathcal{G}_{\epsilon}$ and $\mathcal{P}$. See Figure 4.1 for an example of the set $\mathcal{G}$.

The sets $\mathcal{G}$ and $\mathcal{G}_{\epsilon}$ only differ in the region

$$
\mathcal{N}_{\epsilon}:=\left\{(\lambda, v) \in \mathcal{R}_{\epsilon}:\{\lambda\} \times\left(v_{+}^{*}-\epsilon, v_{+}^{*}+\epsilon\right) \cap \mathcal{G} \neq \emptyset\right\}
$$

The number of connected components of $\mathcal{N}_{\epsilon}$ is finite because the jump $B\left(v_{+}^{*}+\right)-B\left(v_{+}^{*}\right)$ is constant and hence the jumps of $g(\lambda, v)$ are bounded 


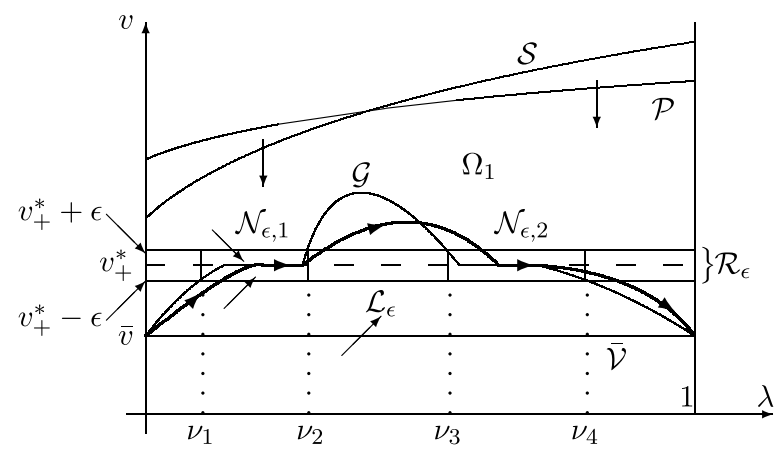

Figure 4.1: The boundary $\mathcal{G}$ and the strips $\mathcal{N}_{\epsilon, 1}, \mathcal{N}_{\epsilon, 2}, \mathcal{L}_{\epsilon}$ when $p_{ \pm}>p_{e}$ and $\mathcal{S} \succ \mathcal{G}$. The thick line is the limit trajectory of $(2.8)$.

away from 0 ; as a consequence, the horizontal length of each connected component of $\mathcal{N}_{\epsilon}$ is bounded away from 0 . For simplicity, we assume in the sequel that

$$
\text { the number of connected components of } \mathcal{N}_{\epsilon} \text { is two, }
$$

as depicted in Figure 4.1. We denote these components by

$$
\begin{aligned}
& \mathcal{N}_{\epsilon, 1}=\left[\nu_{1}, \nu_{2}\right] \times\left(v_{+}^{*}-\epsilon, v_{+}^{*}+\epsilon\right), \\
& \mathcal{N}_{\epsilon, 2}=\left[\nu_{3}, \nu_{4}\right] \times\left(v_{+}^{*}-\epsilon, v_{+}^{*}+\epsilon\right),
\end{aligned}
$$

and the strip between them by

$$
\mathcal{L}_{\epsilon}=\left(\nu_{2}, \nu_{3}\right) \times\left(v_{+}^{*}-\epsilon, v_{+}^{*}+\epsilon\right) .
$$

The results and proofs can be extended to any finite number of connected components.

In the following, when referring to traveling waves, we write for short unique meaning unique up to a shift in $\xi$.

We begin by considering Case (i) under the additional assumption that $\mathcal{S}$ lies above $\mathcal{G}$.

Theorem 4.1. We assume (1.2), (1.4), (2.11), (4.1) and consider end states as in Case (i) of Lemma 2.1, that is, $p_{ \pm}>p_{e}, \lambda_{-}=0$ and $\lambda_{+}=1$; we also assume $\mathcal{G} \prec \mathcal{S}$.

Then, the smoothed traveling-wave problem $(2.8)_{\epsilon}$ has a unique solution $\left(v_{\epsilon}, \lambda_{\epsilon}\right)(\xi)$. Furthermore, the solutions $\left(v_{\epsilon}, \lambda_{\epsilon}\right)(\xi)$ converge uniformly to the unique limit $(v, \lambda)(\xi)$ as $\epsilon \rightarrow 0+$, which is independent of the smoothing. 
At last, the limit $(v, \lambda)(\xi)$ satisfies the end-state conditions $(v, \lambda)( \pm \infty)=$ $\left(\bar{v}, \lambda_{ \pm}\right)$and equation $(2.8)_{2}$ for every $\xi$; equation $(2.8)_{1}$ is satisfied for every $\xi$ such that $v(\xi) \neq v_{+}^{*}$ while when $v(\xi)=v_{+}^{*}$ the differential inclusion

$$
v^{\prime}(\xi) \in\left[\Gamma\left(\lambda(\xi), v_{+}^{*}\right), \Gamma\left(\lambda(\xi), v_{+}^{*}\right)\right]
$$

holds for $\Gamma(\lambda, v)=\frac{g(\lambda, v)}{c s(\lambda, v)}$.

Proof. The relative positions of $\mathcal{G}, \mathcal{S}$ and the end states $(0, \bar{v}),(1, \bar{v})$ are shown in Figure 4.1. In particular, we have $\overline{\mathcal{V}} \prec \mathcal{G}$ in the $\operatorname{strip}(0,1) \times(0, \infty)$. We assume that $\epsilon$ is so small that we also have $\mathcal{G}_{\epsilon} \prec \mathcal{S}$.

The proof of the existence and uniqueness of a solution to $(2.8)_{\epsilon}$ runs as follows, see Theorems 4.1 and 4.3 of [11]. Lemma 3.1 shows that there is an unstable trajectory leaving the point $(0, \bar{v})$ and entering into the region

$$
\Omega_{2}:=\left\{(\lambda, v) \in \Omega: 0<\lambda<1, v>\bar{v}, p>p_{e}, s<0\right\}
$$

More precisely, according to the eigenvector of the eigenvalue $\mu_{2}=(p(\bar{v}, \lambda)-$ $\left.p_{e}\right) / c>0$ given in Lemma 3.1, the trajectory enters into the region $\{(\lambda, v) \in$ $\left.\Omega_{2}: g(\lambda, v)<0\right\}$. Checking the directions of the vector field of $(2.8)_{\epsilon}$ near $\mathcal{P}, \mathcal{S}$ and $\overline{\mathcal{V}}$, we see that they all point inside $\Omega_{2}$. The remaining part of the boundary of $\Omega_{2}$, namely $\lambda=0$ and $\lambda=1$, is formed by solution curves of $(2.8)_{\epsilon}$. Thus, this unstable trajectory cannot exit $\Omega_{2}$ and therefore must go to the other equilibrium point $(1, \bar{v})$. This trajectory $\left(v_{\epsilon}, \lambda_{\epsilon}\right)(\xi)$ is a solution of $(2.8)_{\epsilon}$; moreover, since it stays inside $\Omega_{2}$, then $\lambda(\xi)$ is increasing by the second equation in $(2.8)_{\epsilon}$.

About the uniqueness of the solution of $(2.8)_{\epsilon}$, since $\lambda_{\epsilon}(\xi)$ is monotone we can rewrite the trajectory $\left(v_{\epsilon}, \lambda_{\epsilon}\right)(\xi)$ as $v_{\epsilon}=v_{\epsilon}(\lambda)$. By $(2.8)_{\epsilon}$, one easily sees that $v_{\epsilon}(\lambda)$ satisfies

$$
\left\{\begin{array}{l}
\frac{d v_{\epsilon}}{d \lambda}=\frac{g_{\epsilon}}{s\left(p-p_{e}\right) \lambda(1-\lambda)} \\
v_{\epsilon}(0)=v_{\epsilon}(1)=\bar{v}
\end{array}\right.
$$

and then this is used to prove, as in [11], that $v_{\epsilon}(\lambda)$ is unique.

Now we show the convergence of $\left(v_{\epsilon}, \lambda_{\epsilon}\right)(\xi)$ as $\epsilon \rightarrow 0+$. To this end, we shall show that the family $\left\{\left(v_{\epsilon}, \lambda_{\epsilon}\right)(\xi)\right\}$ is Cauchy for $\epsilon>0$. More precisely, we shall prove that

$$
\left|v_{\epsilon_{1}}(\lambda)-v_{\epsilon_{2}}(\lambda)\right| \leq O(1) \epsilon \quad \text { for } \quad \lambda \in[0,1]
$$


by showing that the inequality in (4.5) holds in each of the intervals $\left[0, \nu_{2}\right]$, $\left[\nu_{2}, \nu_{3}\right],\left[\nu_{3}, \nu_{4}\right]$ and $\left[\nu_{4}, 1\right]$. Here, the term $O(1)$ does not depend on $\epsilon$. Consider $\epsilon_{1}, \epsilon_{2}>0$ and denote

$$
\epsilon:=\max \left\{\epsilon_{1}, \epsilon_{2}\right\} .
$$

We have $g_{\epsilon_{1}}=g_{\epsilon_{2}}=g$ outside the smoothing region $\mathcal{R}_{\epsilon} ;$ then the trajectories

$$
W_{\epsilon_{1}}(\lambda):=\left(\lambda, v_{\epsilon_{1}}(\lambda)\right) \quad \text { and } \quad W_{\epsilon_{2}}(\lambda):=\left(\lambda, v_{\epsilon_{2}}(\lambda)\right)
$$

coincide when they lie outside $\mathcal{R}_{\epsilon}$. Notice that both $W_{\epsilon_{1}}(\lambda)$ and $W_{\epsilon_{2}}(\lambda)$ must enter the strip at the same $\lambda=: \lambda_{0}$, since they coincide before entering the strip. We only have to consider cases where one of the trajectories enters $\mathcal{N}_{\epsilon}$ or $\mathcal{L}_{\epsilon}$.

Case 1. $W_{\epsilon_{1}}$ and $W_{\epsilon_{2}}$ enter $\mathcal{N}_{\epsilon, 1}$.

The direction vectors of (4.4) point downward on the top edge $\mathcal{N}_{\epsilon, 1} \cap\{v=$ $\left.v_{+}^{*}+\epsilon\right\}$ of $\mathcal{N}_{\epsilon, 1}$ while they point upward on the bottom edge $\mathcal{N}_{\epsilon, 1} \cap\{v=$ $\left.v_{+}^{*}-\epsilon\right\}$; as a consequence, once $W_{\epsilon_{1}}$ and $W_{\epsilon_{2}}$ enter $\mathcal{N}_{\epsilon, 1}$, they must exit it through its right edge $\mathcal{N}_{\epsilon, 1} \cap\left\{\lambda=\nu_{2}\right\}$. Thus, we have

$$
\left|v_{\epsilon_{1}}(\lambda)-v_{\epsilon_{2}}(\lambda)\right| \leq 2 \epsilon \quad \text { for } \quad 0 \leq \lambda \leq \nu_{2} .
$$

As $\lambda$ increases in the interval $\left[\nu_{2}, \nu_{3}\right]$, we claim that

$$
\left|v_{\epsilon_{1}}(\lambda)-v_{\epsilon_{2}}(\lambda)\right| \leq O(1) \epsilon \quad \text { for } \quad \nu_{2} \leq \lambda \leq \nu_{3} .
$$

To prove the claim, consider, recalling (4.4),

$$
\begin{aligned}
& \frac{\partial}{\partial v}\left(\frac{g_{\epsilon}}{s\left(p-p_{e}\right) \lambda(1-\lambda)}\right) \\
& \quad=\frac{1}{s^{2}\left(p-p_{e}\right)^{2} \lambda(1-\lambda)}\left[g_{\epsilon, v} s\left(p-p_{e}\right)-g_{\epsilon}\left(s\left(p-p_{e}\right)\right)_{v}\right],
\end{aligned}
$$

where, by (2.10),

$$
g_{\epsilon, v}=B_{\epsilon} c^{2}+B_{\epsilon, v} c^{2}(v-\bar{v})+\left(p_{\lambda v}\left(p-p_{e}\right)+p_{\lambda} p_{v}\right) \lambda(\lambda-1)>0
$$

is bounded away from 0 . Here, we used (2.11), leading to $B_{\epsilon, v} \geq 0$ where $v>\bar{v}$. As $\lambda$ increases in $\left[\nu_{2}, \nu_{2}+o(1)\right]$ range, both $g\left(\lambda, v_{\epsilon_{i}}(\lambda)\right)$ are small because close to the curve $g=0$. Then by (4.8) and (4.9) we see that

$$
\frac{\partial}{\partial v}\left(\frac{g_{\epsilon}}{s\left(p-p_{e}\right) \lambda(1-\lambda)}\right)<0,
$$


for $\lambda \in\left[\nu_{2}, \nu_{2}+o(1)\right]$. Hence, by (4.4), the difference $\left|v_{\epsilon_{1}}(\lambda)-v_{\epsilon_{2}}(\lambda)\right|$ decreases and therefore, by (4.6),

$$
\left|v_{\epsilon_{1}}(\lambda)-v_{\epsilon_{2}}(\lambda)\right| \leq 2 \epsilon \quad \text { for } \quad \nu_{2} \leq \lambda \leq \nu_{2}+o(1),
$$

as $\lambda$ increases until $g_{\epsilon_{1}, v}\left(\lambda, v_{\epsilon_{1}}(\lambda)\right)$ or $g_{\epsilon_{2}, v}\left(\lambda, v_{\epsilon_{2}}(\lambda)\right)$ are bounded away from 0 . Notice that, due to (4.11), one of $g\left(\lambda, v_{\epsilon_{i}}(\lambda)\right)$ being bounded away from 0 implies both are bounded away from 0 .

If at $\lambda=\nu_{2}+o(1)$ both $W_{\epsilon_{i}}(\lambda)$ are already above $\mathcal{L}_{\epsilon}$, then they keep staying above $\mathcal{L}_{\epsilon}$ until $\lambda=\nu_{3}$, because $\mathcal{G}$ is above $\mathcal{L}_{\epsilon}$ and $v_{\epsilon_{i}}(\lambda)$ increase when $W_{\epsilon_{i}}(\lambda)$ are below $\mathcal{G}$. Therefore, over the range $\nu_{2}+o(1) \leq \lambda \leq \nu_{3}$, the functions $v_{\epsilon_{i}}(\lambda)$ satisfy the same ODE (4.4) $)_{1}$, which is independent of $\epsilon_{i}$ 's over that range. By (4.11), the continuous dependence on initial data of regular ODEs implies (4.7)

If instead one of $W_{\epsilon_{i}}\left(\lambda=\nu_{2}+o(1)\right)$ is inside $\mathcal{L}_{\epsilon}$, then, because $g_{\epsilon_{i}, v}(\lambda$, $\left.v_{\epsilon_{i}}(\lambda)\right)$ are bounded away from 0 , it takes another increase in $\lambda$ be $O(1) \epsilon$ for both $W_{\epsilon_{i}}(\lambda)$ to exit $\mathcal{L}_{\epsilon}$ from the top. Over this range $v_{\epsilon_{i}}(\lambda)$ change by $O(1) \epsilon$. Thus, also in this case the estimate (4.7) holds. This completes the proof of the claim (4.7).

We now claim that

$$
\left|v_{\epsilon_{1}}(\lambda)-v_{\epsilon_{2}}(\lambda)\right| \leq O(1) \epsilon \quad \text { for } \quad \nu_{3} \leq \lambda \leq \nu_{4} .
$$

To prove this claim we notice that, over the interval $\nu_{3} \leq \lambda \leq \nu_{4}$, a trajectory $W_{\epsilon_{i}}(\lambda)$ either stays above $\mathcal{N}_{\epsilon, 2}$ for every $\lambda \in\left[\nu_{3}, \nu_{4}\right]$ or enters $\mathcal{N}_{\epsilon, 2}$ at some $\lambda_{\epsilon_{i}, 2} \in\left[\nu_{3}, \nu_{4}\right]$. There are the following three possibilities:

1.1 Both $W_{\epsilon_{1}}(\lambda)$ and $W_{\epsilon_{2}}(\lambda)$ lie above $\mathcal{N}_{\epsilon, 2}$ for every $\lambda \in\left[\nu_{3}, \nu_{4}\right]$.

Then both $v_{\epsilon_{i}}(\lambda)$ satisfy the equation

$$
\frac{d v_{\epsilon_{i}}}{d \lambda}=\frac{g}{s\left(p-p_{e}\right) \lambda(1-\lambda)}
$$

and $\left|v_{\epsilon_{1}}\left(\nu_{3}\right)-v_{\epsilon_{2}}\left(\nu_{3}\right)\right|=O(1) \epsilon$ by (4.7). A straightforward calculation shows that in the region above $\mathcal{G}$ and below $\mathcal{P}$ and $\mathcal{S}, 0 \leq \lambda \leq 1$, the term $\frac{g}{s\left(p-p_{e}\right) \lambda(1-\lambda)}<0$ decreases as $v$ increases. Thus, in this case $\left|v_{\epsilon_{1}}(\lambda)-v_{\epsilon_{2}}(\lambda)\right|$ decreases. This proves (4.12).

1.2 $W_{\epsilon_{1}}(\lambda)$ lies above $\mathcal{N}_{\epsilon, 2}$ for every $\lambda \in\left[\nu_{3}, \nu_{4}\right]$ while $W_{\epsilon_{2}}(\lambda)$ enters $\mathcal{N}_{\epsilon, 2}$ at $\lambda_{\epsilon_{2}, 2} \in\left[\nu_{3}, \nu_{4}\right]$ (or conversely).

As $\lambda$ increases from $\nu_{3}$ and before $W_{\epsilon_{2}}(\lambda)$ enters $\mathcal{N}_{\epsilon, 2}$, the $W_{\epsilon_{i}}$ 's behave as described in Subcase 1.1; then the inequality in (4.12) holds for 
$\lambda \in\left[\nu_{3}, \lambda_{\epsilon_{2}, 2}\right]$. After $W_{\epsilon_{2}}(\lambda)$ enters $\mathcal{N}_{\epsilon, 2}$, it keeps staying inside $\mathcal{N}_{\epsilon, 2}$ because the directions vectors on the top edge and on the bottom edge of $\mathcal{N}_{\epsilon, 2}$ point inside $\mathcal{N}_{\epsilon, 2}$; hence, $v_{\epsilon_{2}}(\lambda)>v_{+}^{*}-\epsilon$. On the other hand, $v_{\epsilon_{1}}(\lambda)$ decreases for all $\lambda \in\left[\nu_{3}, \nu_{4}\right]$. Then (4.12) holds.

1.3 Both $W_{\epsilon_{1}}(\lambda)$ and $W_{\epsilon_{2}}(\lambda)$ enter into $\mathcal{N}_{\epsilon, 2}$ (at $\lambda_{\epsilon_{1}, 2}$ and $\lambda_{\epsilon_{2}, 2}$, respectively).

For $\nu_{3} \leq \lambda \leq \min \left\{\lambda_{\epsilon_{1}, 2}, \lambda_{\epsilon_{2}, 2}\right\}$ we argue as in Subcase 1.1, for $\min \left\{\lambda_{\epsilon_{1}, 2}\right.$, $\left.\lambda_{\epsilon_{2}, 2}\right\} \leq \lambda \leq \max \left\{\lambda_{\epsilon_{1}, 2}, \lambda_{\epsilon_{2}, 2}\right\}$ as in Subcase 1.2. At last, for $\max \left\{\lambda_{\epsilon_{1}, 2}\right.$, $\left.\lambda_{\epsilon_{2}, 2}\right\} \leq \lambda \leq \nu_{4}$ the trajectories cannot exit $\mathcal{N}_{\epsilon, 2}$ until $\lambda$ increases to $\lambda=\nu_{4}$. In this case $\left|v_{\epsilon_{1}}(\lambda)-v_{\epsilon_{2}}(\lambda)\right| \leq 2 \epsilon$ because the height of $\mathcal{N}_{\epsilon, 2}$ is just $2 \epsilon$. This concludes the proof of (4.12).

At last, we claim that

$$
\left|v_{\epsilon_{1}}(\lambda)-v_{\epsilon_{2}}(\lambda)\right| \leq O(1) \epsilon \quad \text { for } \quad \lambda \in\left[\nu_{4}, 1\right] .
$$

To this end, we consider the following three subcases, which possibly occur in subintervals $[a, b]$ of $\left[\nu_{4}, 1\right]$. Recall that every trajectory lies above or inside $\mathcal{N}_{\epsilon, 2}$ at $\lambda=\nu_{4}$, where (4.12) holds; there are no other possibilities. For simplicity, we only discuss the cases where strict inequalities occur (in $\succ, \prec$ and in $d v^{\mathcal{G}} / d \lambda \lessgtr 0$, see below); the remaining possibilities easily follow from our analysis.

1.A Both $W_{\epsilon_{1}}(\lambda) \succ \mathcal{G}_{\epsilon_{1}}$ and $W_{\epsilon_{2}}(\lambda) \succ \mathcal{G}_{\epsilon_{2}}$ for $\lambda \in[a, b] \subset\left[\nu_{4}, 1\right]$. At $\lambda=\nu_{4}$ we have precisely $W_{\epsilon_{1}}\left(\nu_{4}\right) \succ \mathcal{G}_{\epsilon_{1}}$ and $W_{\epsilon_{2}}\left(\nu_{4}\right) \succ \mathcal{G}_{\epsilon_{2}}$, see the warning just above. By the same reasoning in subcase 1.1, the difference $\left|v_{\epsilon_{1}}(\lambda)-v_{\epsilon_{2}}(\lambda)\right|$ decreases as $\lambda$ increases; in this case, the inequality in (4.14) holds in $[a, b]$ if it holds at $a$.

We notice that by the assumptions of this theorem we have $p>p_{e}$ and then $g_{v}>0$; thus, the set $\mathcal{G}$ is a curve of the form $v=v^{\mathcal{G}}(\lambda)$. If the curve $\mathcal{G}$ is decreasing in the sense that $d v^{\mathcal{G}} / d \lambda<0$ in $\left[\nu_{4}, 1\right]$, then no trajectory may intersect $\mathcal{G}$ because at the intersection points the equality $d v_{\epsilon_{i}} / d \lambda=0$ must hold. If this happens, then subcase $1 . A$ occurs over $\left[\nu_{4}, 1\right]$ and (4.14) holds. Therefore, in the rest of the proof of this theorem, we assume that the curve $\mathcal{G}$ is not monotone over $\lambda \in\left[\nu_{4}, 1\right]$.

1.B $W_{\epsilon_{1}}(\lambda) \succ \mathcal{G}_{\epsilon_{1}}$ while $W_{\epsilon_{2}}(\lambda) \prec \mathcal{G}_{\epsilon_{2}}$ (or conversely) for $\lambda \in[a, b] \subset\left[\nu_{4}, 1\right]$. Then $W_{\epsilon_{1}}(\lambda)$ decreases and $W_{\epsilon_{2}}(\lambda)$ increases as $\lambda$ increases. So $\mid v_{\epsilon_{1}}(\lambda)-$ $v_{\epsilon_{2}}(\lambda) \mid$ also decreases as $\lambda$ increases. The inequality in (4.14) holds in $[a, b]$ if it holds at $a$. 
1.C Both $W_{\epsilon_{1}}(\lambda) \prec \mathcal{G}_{\epsilon_{1}}$ and $W_{\epsilon_{2}}(\lambda) \prec \mathcal{G}_{\epsilon_{2}}$ for $\lambda \in[a, b] \subset\left[\nu_{4}, 1\right]$.

In this case both functions $v_{\epsilon_{i}}(\lambda)$ increase with $\lambda$. We further subdivide this case into three subcases as follows.

If both $W_{\epsilon_{i}}(\lambda)$ are inside $\mathcal{R}_{\epsilon}$, then the estimate in (4.14) trivially holds in $[a, b]$. If $W_{\epsilon_{1}}(\lambda)$ is in $\mathcal{R}_{\epsilon}$ while $W_{\epsilon_{2}}(\lambda)$ is outside of $\mathcal{R}_{\epsilon}$ (or conversely), then $v_{\epsilon_{2}}(\lambda)$ increases as $\lambda$ increases. The inequality in (4.14) holds in $[a, b]$ if it holds at $a$.

Then, we are left to the subcase when

$$
\text { both } W_{\epsilon_{i}}(\lambda) \text { are below } \mathcal{R}_{\epsilon} \text {. }
$$

We claim that this cannot happen in a left neighborhood of $\lambda=1$.

To prove the above claim, we observe that the slope of $\mathcal{G}$ for $v \in$ $\left[\bar{v}, v_{+}^{*}-\epsilon\right)$ and $\lambda \in\left(\lambda_{0}, 1\right)$, where $\lambda_{0}<1$ is close enough to 1 , is

$$
\begin{aligned}
\frac{d v^{\mathcal{G}}}{d \lambda} & =-\frac{g_{\lambda}}{g_{v}}=-\frac{\left[p_{\lambda}\left(p-p_{e}\right) \lambda(\lambda-1)\right]_{\lambda}}{B^{\prime} c^{2}(v-\bar{v})+B c^{2}+\left[p_{\lambda}\left(p-p_{e}\right)\right]_{v} \lambda(\lambda-1)} \\
(4.16) & =-\frac{p_{\lambda}\left(p-p_{e}\right)}{B^{\prime} c^{2}(v-\bar{v})+B c^{2}}+O(1)(\lambda-1)<0 .
\end{aligned}
$$

Of course, the choice of $\lambda_{0}$ is independent from $\epsilon$. Since $\mathcal{G}$ is not monotonically decreasing for $\lambda \in\left[\nu_{4}, 1\right]$ but is decreasing for $\lambda$ in a right neighborhood of $\nu_{4}$, there is at least one local minimum point of $\mathcal{G}$, different from 1 , in the interval $\left[\nu_{4}, 1\right]$. Let $\lambda_{1}$ be the maximum of such local minimum points, see Figure 4.2. Then $\lambda_{1}<\lambda_{0}$ holds in view of (4.16). Draw in the $(\lambda, v)$-plane a horizontal line $\mathcal{H}$ through the minimum of $\mathcal{G}$ over the interval $\nu_{4} \leq \lambda \leq 1$. Since $g<0$ when $v=\bar{v}$ and $\lambda \in(0,1)$, the line $\mathcal{H}$ is strictly contained between the lines $v=v_{+}^{*}$ and $v=\bar{v}$; hence, the line $\mathcal{H}$ intersects $\mathcal{G}$ to the right of $\lambda_{1}$. Denote the $\lambda$-coordinate of the rightmost of such intersection points as $\lambda_{2}$. Clearly, we have $\lambda_{2}<1$ and $\lambda_{2}$ is independent of $\epsilon$.

As $\lambda$ increases from $\nu_{4}$ towards 1 , both $W_{\epsilon_{1}}(\lambda)$ and $W_{\epsilon_{2}}(\lambda)$ start from above the line $v=v_{+}^{*}-\epsilon$. We recall that when a trajectory $W_{\epsilon}$ is under $\mathcal{G}$, then its component $v_{\epsilon}(\lambda)$ increases. If a trajectory lie below $\mathcal{G}$ at $\lambda=\lambda_{2}$, then it must have been increasing in the interval $\left[\nu_{4}, \lambda_{2}\right]$ and then it could not start from above the line $v=v_{+}^{*}-\epsilon$. As a consequence, at $\lambda=\lambda_{2}$ both $W_{\epsilon_{1}}(\lambda)$ and $W_{\epsilon_{2}}(\lambda)$ must lie above $\mathcal{G}$. This means, on the one hand, that case 1 . $A$ occurs in the region $\lambda_{2} \leq \lambda \leq 1$; on the other hand, the intervals over which (4.15) possibly occurs must be on the right side of $\lambda_{2}$. This proves the claim above. 


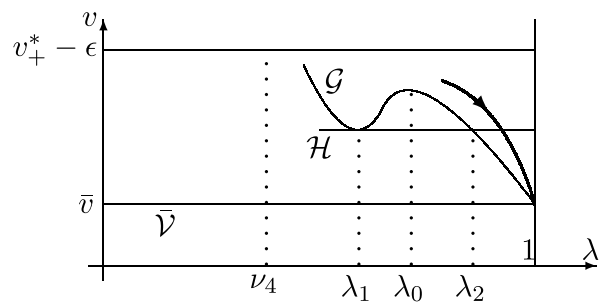

Figure 4.2: The boundary $\mathcal{G}$ and the line $\mathcal{H}$.

To conclude the proof, we remark that in this case both $v_{\epsilon_{i}}(\lambda)$ satisfy the same regular ODE (4.13). We argue as for proving (4.10), by exploiting $\lambda_{2}$ to deduce a strictly negative bound of (4.10), which is moreover independent of $\epsilon$ : if the inequality in (4.14) is true at $a$ then it is still true for $a \leq \lambda \leq \min \left\{\lambda_{2}, b\right\}$.

This completely proves claim (4.14).

Combining (4.6), (4.7), (4.12) and (4.14) we conclude that (4.5) is satisfied in Case 1.

Case 2. $W_{\epsilon_{1}}(\lambda)$ and $W_{\epsilon_{2}}(\lambda)$ do not enter $\mathcal{N}_{\epsilon, 1}$ but enter $\mathcal{L}_{\epsilon}$. The proof of Case 1 also covers this case.

Case 3. $W_{\epsilon_{1}}(\lambda)$ and $W_{\epsilon_{2}}(\lambda)$ enter neither $\mathcal{N}_{\epsilon, 1}$ nor $\mathcal{L}_{\epsilon}$ but enter $\mathcal{N}_{\epsilon, 2}$. Again, the proof of Case 1 also covers this case.

Thus, estimate (4.5) holds for all cases. This proves that

$$
\lim _{\epsilon \rightarrow 0+} v_{\epsilon}(\lambda)=v(\lambda) \quad \text { in } \quad\|\cdot\|_{C([0,1])} \cdot
$$

Furthermore, in the above argument the smoothing of $B$ is arbitrary as long as the width of region of smoothing is $\epsilon$ and the smoothing is monotone preserving. Hence, estimate (4.5) is independent of the smoothing of $B$ and therefore the limit $v(\lambda)$ is unique in the sense that it is independent of the smoothing.

It remains to prove that the limit $(v, \lambda)(\xi)$ is a solution as stated in the theorem. When $v(\xi) \neq v_{ \pm}^{*}$, being the strong limit of solutions $\left(v_{\epsilon}, \lambda_{\epsilon}\right)(\xi)$ of $(2.8)_{\epsilon},(v, \lambda)(\xi)$ is a strong solution of (2.8). When $\xi$ is in the interior of the set $\left\{\xi: v(\xi)=v_{ \pm}^{*}\right\}$, then $v^{\prime}(\xi)=0$. Notice that $\Gamma\left(\lambda(\xi), v_{+}^{*}\right)>0$ and $\Gamma\left(\lambda(\xi), v_{+}^{*}+\right)<0$, we conclude that (4.3) is true. This concludes the proof of the theorem.

Corollary 4.2. We assume (1.2), (1.3), (1.4), (2.11), (4.1) and consider end states as in Case (i) of Lemma 2.1, that is, $p_{ \pm}>p_{e}, \lambda_{-}=0$ and $\lambda_{+}=1$. 
Then, for small enough $c>0$, problem $(2.8)_{\epsilon}$ has a unique solution in the sense of the vanishing smoothing.

Proof. When $p_{ \pm}>p_{e}$ the curve $\mathcal{P}$ is above $\mathcal{G}$. By the physical assumptions (1.3) on the pressure we have

$$
\lim _{c \rightarrow 0+} v^{\mathcal{S}}(\lambda)=\infty
$$

Therefore $\mathcal{S}$ lies above $\mathcal{P}$ and hence $\mathcal{G}$ for small enough $c>0$. Then, Theorem 4.1 applies.

We pursue the analysis of Case $(i)$ by considering the case when $\mathcal{S}$ lies below $\mathcal{G}$. The following statements parallel that of Theorem 4.1; for brevity, from here on we stress any more neither that our solutions to (2.8) in the sense of the vanishing smoothing are independent of the smoothing, as long as (2.11) holds, nor we state the properties of the solutions as in the last part of the statement of Theorem 4.1 .

Theorem 4.3. We assume (1.2), (1.4), (2.11), (4.1) and consider end states as in Case (i) of Lemma 2.1, that is, $p_{ \pm}>p_{e}, \lambda_{-}=0$ and $\lambda_{+}=1$; we also assume $\mathcal{G} \succ \mathcal{S}$.

Then, the traveling-wave problem (2.8) has a unique solution $(v, \lambda)(\xi)$ in the sense of the vanishing smoothing.

Proof. The proof for this theorem is almost the same as that of Theorem 4.1, except that instead of tracing the trajectories in the $\lambda$ increasing direction, we trace the trajectories in the $\lambda$ decreasing direction in this proof. See Figure 4.3.

Corollary 4.4. We assume (1.2), (1.3), (1.4), (2.11), (4.1) and consider end states as in Case (i) of Lemma 2.1, that is, $p_{ \pm}>p_{e}, \lambda_{-}=0$ and $\lambda_{+}=1$.

Then, for large enough $c>0$, problem $(2.8)_{\epsilon}$ has a unique solution in the sense of the vanishing smoothing.

Proof. When $c>0$ is large enough the curve $\mathcal{S}$ lies below $\overline{\mathcal{V}}$, and hence Theorem 4.3 applies.

We conclude the analysis of Case (i) by considering the case when $\mathcal{S}$ intersects $\mathcal{G}$.

Theorem 4.5. We assume (1.2), (1.4), (2.11), (4.1) and consider end states as in Case (i) of Lemma 2.1, that is, $p_{ \pm}>p_{e}, \lambda_{-}=0$ and $\lambda_{+}=1$; moreover, we assume that $\mathcal{S}$ and $\mathcal{G}$ intersect transversely only once. 


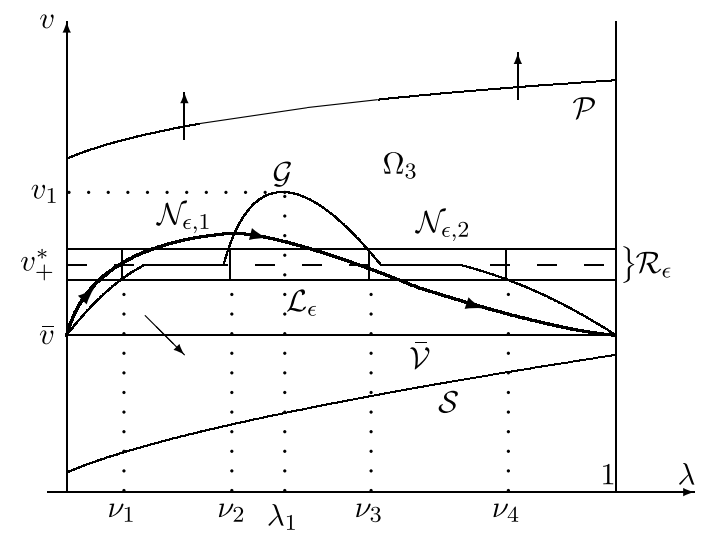

Figure 4.3: The boundary $\mathcal{G}$ and the strips $\mathcal{N}_{\epsilon, 1}, \mathcal{N}_{\epsilon, 2}, \mathcal{L}_{\epsilon}$ when $p_{ \pm}>p_{e}$ and $\mathcal{S} \prec \mathcal{G}$.

Then, the traveling-wave problem (2.8) has a unique solution $(v, \lambda)(\xi)$ in the sense of the vanishing smoothing.

Proof. Consider the smoothed system $(2.8)_{\epsilon}$. We denote by $\left(\lambda_{0}, v_{0}\right)$ the intersection point of $\mathcal{S}$ and $\mathcal{G}$. Since $\mathcal{S}$ and $\mathcal{G}$ intersect transversely, also the sets $\mathcal{S}$ and $\mathcal{G}_{\epsilon}$ still intersect once. Denote their point of intersection as $\left(\lambda_{0}^{\epsilon}, v_{0}^{\epsilon}\right)$.

If $\left(\lambda_{0}, v_{0}\right)$ (or $\left.\left(\lambda_{0}^{\epsilon}, v_{0}^{\epsilon}\right)\right)$ is outside of the strip $\mathcal{R}_{\epsilon}$, then $\left(\lambda_{0}^{\epsilon}, v_{0}^{\epsilon}\right)=\left(\lambda_{0}, v_{0}\right)$.

If $\left(\lambda_{0}, v_{0}\right)$ lies inside the strip $\mathcal{R}_{\epsilon}$, then $\left(\lambda_{0}^{\epsilon}, v_{0}^{\epsilon}\right)$ is also inside of $\mathcal{R}_{\epsilon}$. Note that the set $\mathcal{S} \cap \mathcal{R}_{\epsilon}$ has a radius $O(1) \epsilon$ : the curve $\mathcal{S}$ intersects the line $v=v_{+}^{*}$ transversely because the function $v^{\mathcal{S}}$ is strictly increasing. Then the intersection point $\left(\lambda_{0}^{\epsilon}, v_{0}^{\epsilon}\right) \in \mathcal{S} \cap \mathcal{R}_{\epsilon}$, must satisfy

$$
\left|\lambda_{0}^{\epsilon}-\lambda_{0}\right|+\left|v_{0}^{\epsilon}-v_{0}\right|=O(1) \epsilon
$$

Then, as $\epsilon \rightarrow 0+,\left(\lambda_{0}^{\epsilon}, v_{0}^{\epsilon}\right)$ goes to the point of intersection $\left(\lambda_{0}, v_{0}\right)$ of $\mathcal{S}$ and $\mathcal{G}$.

According to Theorems 4.1 and 4.3 of [11], there is a unique solution $\left(v_{\epsilon}, \lambda_{\epsilon}\right)(\xi)$ for the problem $(2.8)_{\epsilon}$; moreover, this solution passes through the point $\left(\lambda_{0}^{\epsilon}, v_{0}^{\epsilon}\right)$.

The solution is constructed this way: Through the point $\left(\lambda_{0}^{\epsilon}, v_{0}^{\epsilon}\right)$, there are two trajectories of $(2.8)_{\epsilon}$, see the proof of Theorem 4.1 of [11]. One of them goes through $\left(\lambda_{0}^{\epsilon}, v_{0}^{\epsilon}\right)$ from the region

$$
\Omega_{4}:=\left\{(\lambda, v) \in \Omega: 0 \leq \lambda<\lambda_{0}, g_{\epsilon}>0, p>p_{e}, s>0\right\}
$$




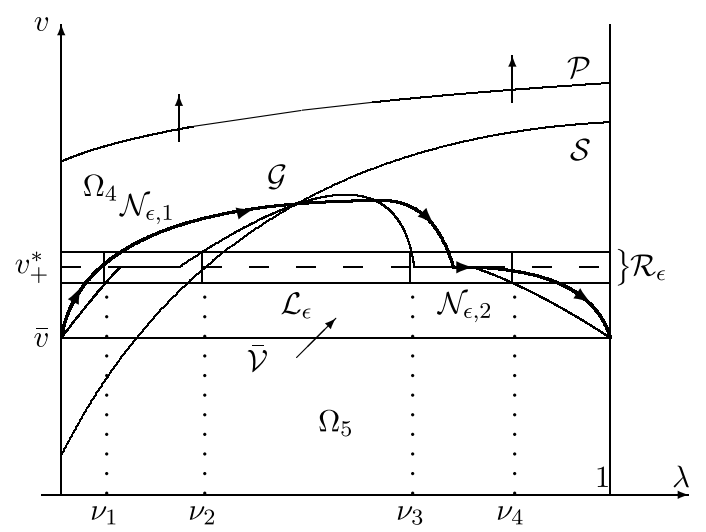

Figure 4.4: The boundary $\mathcal{G}$ and the strips $\mathcal{N}_{\epsilon, 1}, \mathcal{N}_{\epsilon, 2}, \mathcal{L}_{\epsilon}$ when $p_{ \pm}>p_{e}$ and $\mathcal{S}$ intersects $\mathcal{G}$.

to the region

$$
\Omega_{5}:=\left\{(\lambda, v) \in \Omega: \lambda_{0}<\lambda \leq 1, g_{e}<0, p>p_{e}, s<0\right\}
$$

see Figure 4.4.

To show that $\left\{\left(v_{\epsilon}, \lambda_{\epsilon}\right)\right\}_{\epsilon>0}$ is Cauchy we consider solutions $\left(v_{\epsilon_{i}}, \lambda_{\epsilon_{i}}\right)(\xi)$ of $(2.8)_{\epsilon_{i}}$ for $i=1,2$. When we trace the trajectory from $\left(\lambda_{0}^{\epsilon}, v_{0}^{\epsilon}\right)$ back towards $(0, \bar{v})$, the situation is the same as that in Theorem 4.3. On the other hand, when we follow the trajectory forwards from $\left(\lambda_{0}^{\epsilon}, v_{0}^{\epsilon}\right)$ towards $(1, \bar{v})$, we encounter the same situation described in proof of Theorem 4.1. Combining with $(4.17)$, we see that $\left\{\left(v_{\epsilon}, \lambda_{\epsilon}\right)\right\}$ is Cauchy and hence the desired conclusion holds.

The degeneracy encountered in Theorem 4.5 occurs also in other papers. A suggestive terminology is introduced in [38], where they call the curve $\mathcal{S}$ the wall of singularities or singular barrier, while the intersection point $\mathcal{S} \cap \mathcal{G}$ is called the hole in the wall: it is through the hole that the trajectory can pass beyond the wall. Related papers are [38, 34]; an extension to a $3 \times 3$ model is done in [35] and a rigorous proof is given in [27]. Another example occurs in modeling the spreading of pollution $[6,12]$.

Remark 4.1. A discussion about the structure the vanishing smoothing solution is now in order. From the proofs of Theorems 4.1 and 4.3, we can see that the vanishing smoothing solution of (2.8) can be constructed as follows; we use the end states in Theorem 4.1 as an example, see Figure 4.1. 
Consider the unique unstable trajectory of (2.8), without smoothing, issued from $(0, \bar{v})$ into the $\{\lambda>0\}$ region. As $\xi$ increases from $-\infty, \lambda(\xi)$ increases from $\lambda=0$. If this trajectory meets

$$
\mathcal{N}:=\mathcal{G} \cap\left\{v=v_{ \pm}^{*}\right\},
$$

say at $\lambda=\lambda_{1}$, then the classical trajectory ends there. On the contrary, the vanishing smoothing trajectory continues by following $\mathcal{N}$ horizontally in $(\lambda, v)$-plane as $(\lambda, v)(\xi)=\left(\lambda(\xi), v_{+}^{*}\right)$, where $\lambda(\xi)$ is determined by $(2.8)_{2}$ with $v=v_{+}^{*}$. This is because the flow direction of $(2.8)_{\epsilon}$, as we are tracing forward, points towards $\mathcal{N}_{\epsilon}$. This nonclassical branch terminates when $\mathcal{G}$ deviates from $v=v_{+}^{*}$, say at $\lambda_{2}=\lambda\left(\xi_{2}\right)$. As $\xi$ increases from $\xi_{2}$, the trajectory follows the solution of (2.8) with initial value at $\xi=\xi_{2}$ given by $(\lambda, v)\left(\xi_{2}\right)=\left(\lambda_{2}, v_{+}^{*}\right)$. Then, the overall structure of the vanishing smoothing trajectory consists of pieces of constant $v=v_{+}^{*}$ or $v=v_{-}^{*}$ segments, connected by regular trajectories of (2.8).

More precisely, assume that the proof of existence of solutions to $(2.8)_{\epsilon}$ is made by following forwards an unstable trajectory to the other equilibrium point and, moreover, that the trajectory of $(2.8)_{\epsilon}$ can exit a connected component of $\mathcal{N}_{\epsilon}$ only at its $\lambda=$ constant ends; then, the constant pieces of the limit solution to (2.8) terminate at the right end of the connected components of $\mathcal{N}$, as $\xi$ increases. On the other hand, assume that the existence proof is made by tracing backwards a stable trajectory to the other equilibrium point, and that the flow direction of (2.8), as we are tracing backwards, points towards $\mathcal{N}_{\epsilon}$. Then the trajectory can exit a connected component of $\mathcal{N}_{\epsilon}$ only at its $\lambda=$ constant boundary; then the constant pieces of the limit solution to (2.8) start at a left end of a connected component of $\mathcal{N}$, as $\xi$ increases.

In the sequel, we also call vanishing smoothing solutions the solutions of (2.8) with discontinuous $B(v)$ constructed in this way, bypassing the limit procedure.

Remark 4.2. We can extend Theorems 4.1, 4.3 and 4.5 to include the case where $p_{-}=p_{e}$ and $\lambda_{-}=0$. The method consists in selecting a sequence $\left\{\bar{v}_{n}\right\}$ so that $\bar{v}_{n} \rightarrow \bar{v}+$. Then $p\left(\lambda_{-}=0, \bar{v}_{n}\right)>p_{e}$ and hence the theorems quoted above apply and provide traveling waves $\left(v_{n}, \lambda_{n}\right)(\xi)$. By the same argument exploited in the proof of Corollary 4.2 in [10], we can show that $\left(v_{n}, \lambda_{n}\right)(\xi)$ converges, by extracting a subsequence if necessary, to a nonconstant limit $(v, \lambda)(\xi)$. This limit satisfies the ODEs in $(2.8)$ weakly, and hence strongly when $v(\xi) \neq v_{ \pm}^{*}$. 
By Remark 4.1 the structure of each trajectory $\left(v_{n}, \lambda_{n}\right)(\xi)$ consists of finitely many line segments lying on $v=v_{ \pm}^{*}$ that are joined by trajectories of the dynamical system in (2.8). The number of pieces is bounded independently from $n$. Then the limit $(v, \lambda)(\xi)$ will have the same structure and hence is a solution of (2.8) in the sense of the vanishing smoothing. Furthermore, the uniqueness of the solution can be proved almost as in Corollary 4.2 of $[10]$.

\section{Existence and convergence of solutions $\left(v_{\epsilon}, \lambda_{\epsilon}\right)(\xi)$, Cases (ii) $-(v)$}

In this section we conclude the proof of Theorem 4.1 by considering Cases (ii) $-(v)$. We begin with Case (ii).

Theorem 5.1. We assume (1.2), (1.4), (2.11), (4.1) and consider end states as in Case (ii), that is, $p_{ \pm}<p_{e}, \lambda_{-}=1$ and $\lambda_{+}=0$. Moreover, we assume that $\mathcal{S}$ and $\mathcal{G}$ do not intersect.

Then, the traveling-wave problem (2.8) has a unique solution $(v, \lambda)(\xi)$ in the sense of the vanishing smoothing if

$$
B c^{2}+\left(p_{\lambda v}\left(p-p_{e}\right)+p_{\lambda} p_{v}\right) \lambda(\lambda-1)>0,
$$

for $v$ near $v_{-}^{*}$.

Proof. We notice that the assumption $\mathcal{S} \cap \mathcal{G}=\emptyset$ implies that either $s_{ \pm}>0$ or $s_{ \pm}<0$ holds. We only provide a sketch of the existence proof, which is similar to that of Theorem 4.4 in [11] with the modifications introduced for the vanishing smoothing in the proofs of the previous section.

Case 1: $s_{ \pm}<0$.

In this case we have $\mathcal{P} \prec \mathcal{G} \prec \overline{\mathcal{V}} \prec \mathcal{S}$, see Figure 5.1(1), where we omitted to draw $\mathcal{G}$ for simplicity. The flow directions of $(2.8)_{\epsilon}$ point inside the set

$$
\Omega_{6}:=\left\{(\lambda, v) \in \Omega: v<\bar{v}, p<p_{e}\right\}
$$

at points inside and near the boundaries $\mathcal{S}$ and $\mathcal{P}$; the remaining parts of the boundary of $\Omega_{6}$, namely $\lambda=0$ and $\lambda=1$, are solution curves of $(2.8)_{\epsilon}$. Therefore, the trajectories of $(2.8)_{\epsilon}$ cannot leave $\Omega_{6}$ once they entered it. By Lemma 3.1 there is an unstable manifold of $\left(\lambda_{-}=1, \bar{v}\right)$ entering $\Omega_{6}$. Following this unstable trajectory forwards, we see that it must connect to the equilibrium point $\left(\lambda_{+}=0, \bar{v}\right)$. Thus, a solution of $(2.8)_{\epsilon}$ exists in this case. Moreover, according to Theorem 4.5 of [11], this solution is unique. 


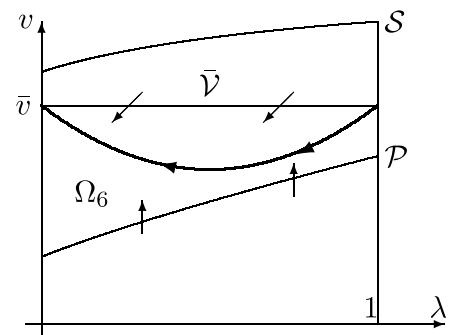

(1)

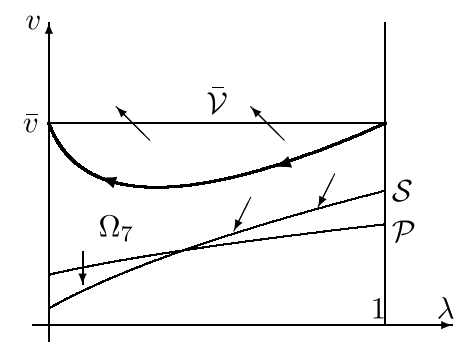

(2)

Figure 5.1: The cases $s_{ \pm}<0$, left, and $s_{ \pm}>0$, right.

We claim that the trajectories of $(4.4)_{\epsilon}$ can exit $\mathcal{N}_{\epsilon}$ at a $\lambda=$ constant end as $\xi$ increases. This is because, below the line $v=v_{-}^{*}$, the larger $B$ for turbulent flow is in effect, while $B$ is smaller above $v=v_{-}^{*}$, see Figure 2.1; in other words, $B\left(v_{-}^{*}\right)>B\left(v_{-}^{*}+\right)$. Since $v_{-}^{*}<\bar{v}$, we have

$$
g\left(\lambda, v_{-}^{*}+\right)=B\left(v_{-}^{*}+\right) c^{2}\left(v_{-}^{*}-\bar{v}\right)+\left.p_{\lambda}\left(p-p_{e}\right)\right|_{v=v_{-}^{*}+} \lambda(\lambda-1)>g\left(\lambda, v_{-}^{*}-\right) .
$$

Because $g$ changes sign across $\mathcal{N}$, then the inequality

$$
g\left(\lambda, v_{-}^{*}+\right)>0>g\left(\lambda, v_{-}^{*}-\right)
$$

holds across the line segments of $\mathcal{N}$. Then, below $\mathcal{N}_{\epsilon}$ the directions of $(2.8)_{\epsilon}$ are upwards while above $\mathcal{N}$ the directions of $(2.8)_{\epsilon}$ are downwards. This shows that the claim holds.

By the technical assumption (5.1) we deduce

$$
B_{\epsilon} c^{2}+\left(p_{\lambda v}\left(p-p_{e}\right)+p_{\lambda} p_{v}\right) \lambda(\lambda-1)>0
$$

over $\left(v_{-}^{*}-\delta, v_{-}^{*}+\delta\right)$ for some small $\delta>0$. Then, we have

$$
g_{\epsilon, v}=B_{\epsilon} c^{2}+\left(p_{\lambda v}\left(p-p_{e}\right)+p_{\lambda} p_{v}\right) \lambda(\lambda-1)+B_{\epsilon, v}(v) c^{2}(v-\bar{v})>0
$$

for $v \in\left(v_{-}^{*}-\delta, v_{-}^{*}+\delta\right)$. As in the calculation of (4.8), we have

$$
\frac{\partial}{\partial v}\left(\frac{g_{\epsilon}}{s\left(p-p_{e}\right) \lambda(1-\lambda)}\right)>0
$$

for $\lambda$ near the left ends of the connected components of $\mathcal{N}_{\epsilon}$. 


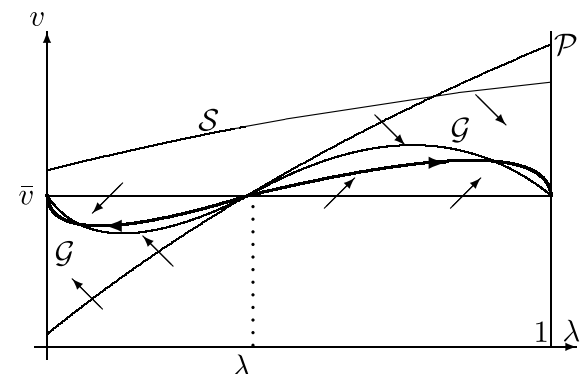

(a)

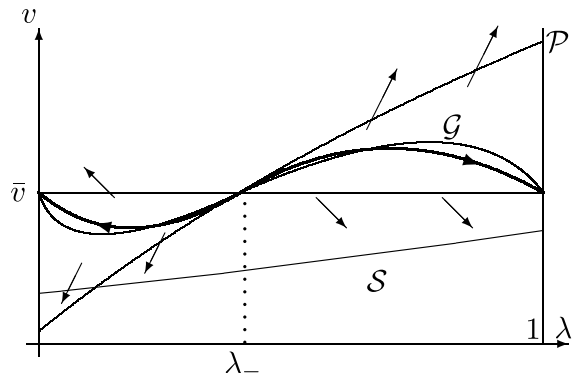

(b)

Figure 5.2: The case $p_{-}=p_{e} .(a)$ : subcase $s_{ \pm}<0,(b)$ : subcase $s_{ \pm}>0$. The rightward trajectories refer to Case (iii), the leftward ones to Case (iv).

Thus, arguing as in the proofs of Theorems 4.1, except that we trace the trajectory in the $\lambda$ decreasing direction, we conclude that there is a unique solution of (2.8) in the sense of vanishing smoothing.

Case 2: $s_{ \pm}>0$.

The proof of this case is analogous to that of Case 1, with the difference that now we trace the stable trajectory of the equilibrium point $(0, \bar{v})$ backwards; see Figure 5.1(2). The details are left to the reader.

Corollary 5.2. We assume (1.2), (1.3), (1.4), (2.11), (4.1) and consider end states as in Case (ii), i.e., $p_{ \pm}<p_{e}, \lambda_{-}=1$ and $\lambda_{+}=0$.

Then, both for large enough $c>0$, problem (2.8) has a unique solution in the sense of the vanishing smoothing.

Now we investigate Cases (iii) and (iv): the pressure at an end state equals $p_{e}$.

Theorem 5.3. We assume (1.2), (1.4), (2.11), (4.1) and consider end states either as in Case (iii), i.e., $p_{+}>p_{e}=p_{-}$and $\lambda_{-}<1=\lambda_{+}$, or as in Case (iv), i.e., $p_{+}<p_{e}=p_{-}$and $\lambda_{-}>0=\lambda_{+}$. Moreover, in Case (iii) we assume that $\mathcal{S}$ and $\mathcal{G}$ intersect transversally at most once in the region $\left(\lambda_{-}, 1\right] \times(0, \infty)$ while in Case (iv) we assume that they do not intersect at all.

Then, the traveling-wave problem (2.8) has a unique solution $(v, \lambda)(\xi)$ in the sense of the vanishing smoothing.

Proof. We consider both Case (iii) and Case (iv) at the same time; the analysis is divided into three subcases according to the position of the curve $\mathcal{S}$. The relative positions of the sets $\mathcal{S}, \mathcal{G}$ and $\mathcal{P}$ are shown in Figure 5.2.

Case 1. $s_{ \pm}<0$. 
By Lemma 3.1, there is an unstable trajectory of the equilibrium $\left(\lambda_{-}, \bar{v}\right)$ entering the $\lambda>\lambda_{-}$side (Case (iii)) and the $\lambda<\lambda_{-}$side (Case (iv)), respectively. On the $\lambda>\lambda_{-}$side, the trajectory is necessarily below $\mathcal{P}$ at the beginning: otherwise, we should have $\lambda^{\prime}<0$, a contradiction. Then, the situation on the $\lambda>\lambda_{-}$side is analogous to that of the proof of Theorem 4.1. On the other hand, the situation in the other side $\lambda<\lambda_{-}$is similar to that of Case 1 in the proof of Theorem 5.1. This shows the existence and uniqueness of the vanishing smoothing solution to (2.8) in the case $s_{ \pm}<0$.

Case 2. $s_{ \pm}>0$.

Under the assumptions we made on the sets $\mathcal{S}$ and $\mathcal{G}$, the situation in the side $\lambda>\lambda_{-}$is analogous to that in the proof of Theorem 4.3, while in the side $\lambda<\lambda_{-}$it is similar to that of Case 2 in the proof of Theorem 5.1. The arguments used there can be used here as well.

Case 3. $s_{+}<0<s_{-}$and $p_{+}>p_{e}=p_{-}, \lambda_{-}<1=\lambda_{+}$.

The proof is similar to that of Theorem 4.5.

Remark 5.1. Theorem 5.1 can be extended to include the case where $\lambda_{-}=1$, $p_{-}=p_{e}$. The proof is analogous to that outlined in Remark 4.2.

Now, combining Theorems 4.1, 4.3, 4.5 and Remark 4.2, we get statement (i) of Theorem 2.2. Similarly, statement (ii) of Theorem 2.2 is the combination of Theorem 5.1 and Remark 4.2. Statements (iii) and (iv) of Theorem 2.2 is deduced by Theorem 5.3 and Remark 5.1. At last, statement (v) in Theorem 2.2 is easily discussed as in Lemma 5.3 of [11].

\section{References}

[1] D. Amadori and A. Corli. On a model of multiphase flow. SIAM J. Math. Anal., 40(1):134-166, 2008. MR2403315

[2] D. Amadori and A. Corli. Global existence of BV solutions and relaxation limit for a model of multiphase reactive flow. Nonlinear Anal., 72(5):2527-2541, 2010. MR2577817

[3] D. Amadori and A. Corli. Solutions for a hyperbolic model of multiphase flow. In Proceedings of the Conference "Multiphase flow in industrial and environmental engineering". Springer, 2013.

[4] D. Amadori, L. Gosse, and G. Guerra. Godunov-type approximation for a general resonant balance law with large data. J. Differential Equations, 198(2):233-274, 2004. MR2038581 
[5] F. Asakura and A. Corli. Global existence of solutions by path decomposition for a model of multiphase flow. Quart. Appl. Math., 71:135182, 2013. MR3075539

[6] E. Barbera, C. Currò, and G. Valenti. A hyperbolic model for the effects of urbanization on air pollution. Appl. Math. Model., 34(8):2192-2202, 2010. MR2607751

[7] H. Berestycki, B. Nicolaenko, and B. Scheurer. Traveling wave solutions to combustion models and their singular limits. SIAM J. Math. Anal., 16(6):1207-1242, 1985. MR0807905

[8] A. Corli and H. Fan. The Riemann problem for reversible reactive flows with metastability. SIAM J. Appl. Math., 65(2):426-457, 2004/05. MR2123064

[9] A. Corli and H. Fan. A hyperbolic model for phase transitions in porous media. In F. Ancona, A. Bressan, P. Marcati, and A. Marson, editors, Hyperbolic Problems: Theory, Numerics, Applications. Proceedings of the $14^{\text {th }}$ Int. Conf. on Hyperbolic Problems. Amer. Inst. Math. Sc., 2012.

[10] A. Corli and H. Fan. Traveling waves in porous media with phasedependent damping. Preprint, 2013.

[11] A. Corli and H. Fan. Traveling waves of phase transitions in porous media. Appl. Anal., 92:1217-1240, 2013.

[12] C. Currò and D. Fusco. Discontinuous travelling wave solutions for a class of dissipative hyperbolic models. Atti Accad. Naz. Lincei Cl. Sci. Fis. Mat. Natur. Rend. Lincei (9) Mat. Appl., 16(1):61-71, 2005. MR2225923

[13] C. M. Dafermos. A system of hyperbolic conservation laws with frictional damping. Z. Angew. Math. Phys., 46(Special Issue):S294-S307, 1995. MR1359325

[14] C. M. Dafermos and R. Pan. Global $B V$ solutions for the $p$-system with frictional damping. SIAM J. Math. Anal., 41(3):1190-1205, 2009. MR2529961

[15] H. Fan. Traveling waves, Riemann problems and computations of a model of the dynamics of liquid/vapor phase transitions. J. Differential Equations, 150(2):385-437, 1998. MR1658672

[16] H. Fan. On a model of the dynamics of liquid/vapor phase transitions. SIAM J. Appl. Math., 60(4):1270-1301, 2000. MR1760035 
[17] H. Fan. Symmetry breaking, ring formation and other phase boundary structures in shock tube experiments on retrograde fluids. J. Fluid Mech., 513:47-75, 2004. MR2259785

[18] H. Fan and X.-B. Lin. Collapsing and explosion waves in phase transitions with metastability, existence, stability and related Riemann problems. J. Dynam. Differential Equations, 22(2):163-191, 2010. MR2665432

[19] H. Fan and X.-B. Lin. Standing waves for phase transitions in a spherically symmetric nozzle. SIAM J. Math. Anal., 44(1):405-436, 2012. MR2888294

[20] H. Fan and M. Slemrod. Dynamic flows with liquid/vapor phase transitions. In Handbook of mathematical fluid dynamics, Vol. I, pages 373-420. North-Holland, Amsterdam, 2002. MR1942467

[21] A. F. Filippov. Differential equations with discontinuous righthand sides. Kluwer Academic Publishers Group, Dordrecht, 1988. MR1028776

[22] P. Gordon. Recent mathematical results on combustion in hydraulically resistant porous media. Math. Model. Nat. Phenom., 2(2):56-76, 2007. MR2445587

[23] G. Guerra. Well-posedness for a scalar conservation law with singular nonconservative source. J. Differential Equations, 206(2):438-469, 2004. MR2095821

[24] B. Hanouzet and R. Natalini. Global existence of smooth solutions for partially dissipative hyperbolic systems with a convex entropy. Arch. Ration. Mech. Anal., 169(2):89-117, 2003. MR2005637

[25] J. Hong and B. Temple. The generic solution of the Riemann problem in a neighborhood of a point of resonance for systems of nonlinear balance laws. Methods Appl. Anal., 10(2):279-294, 2003. MR2074752

[26] J. Hong and B. Temple. A bound on the total variation of the conserved quantities for solutions of a general resonant nonlinear balance law. SIAM J. Appl. Math., 64(3):819-857, 2004. MR2068124

[27] H. Hoshino. Traveling wave analysis for a mathematical model of malignant tumor invasion. Analysis (Munich), 31(3):237-248, 2011. MR2822308 
[28] L. Hsiao and T.-P. Liu. Convergence to nonlinear diffusion waves for solutions of a system of hyperbolic conservation laws with damping. Comm. Math. Phys., 143(3):599-605, 1992. MR1145602

[29] L. Hsiao and D. Serre. Global existence of solutions for the system of compressible adiabatic flow through porous media. SIAM J. Math. Anal., 27(1):70-77, 1996. MR1373147

[30] F. Huang, R. Pan, and Z. Wang. $L^{1}$ convergence to the Barenblatt solution for compressible Euler equations with damping. Arch. Ration. Mech. Anal., 200(2):665-689, 2011. MR2787593

[31] E. Isaacson and B. Temple. Convergence of the $2 \times 2$ Godunov method for a general resonant nonlinear balance law. SIAM J. Appl. Math., 55(3):625-640, 1995. MR1331577

[32] M. Luskin. On the existence of global smooth solutions for a model equation for fluid flow in a pipe. J. Math. Anal. Appl., 84(2):614-630, 1981. MR0639688

[33] M. Luskin and B. Temple. The existence of a global weak solution to the nonlinear waterhammer problem. Comm. Pure Appl. Math., 35(5):697-735, 1982. MR0668411

[34] B. P. Marchant, J. Norbury, and A. J. Perumpanani. Travelling shock waves arising in a model of malignant invasion. SIAM J. Appl. Math., 60(2):463-476 (electronic), 2000. MR1740255

[35] B. P. Marchant, J. Norbury, and J. A. Sherratt. Travelling wave solutions to a haptotaxis-dominated model of malignant invasion. Nonlinearity, 14(6):1653-1671, 2001. MR1867097

[36] T. Nishida. Nonlinear hyperbolic equations and related topics in fluid dynamics. Département de Mathématique, Université de Paris-Sud, Orsay, 1978. Publications Mathématiques d'Orsay, No. 78-02. MR0481578

[37] R. Pan. Darcy's law as long-time limit of adiabatic porous media flow. J. Differential Equations, 220(1):121-146, 2006. MR2182082

[38] G. Pettet, D. McElwain, and J. Norbury. Lotka-volterra equations with chemotaxis; walls, barriers and travelling waves. IMA J. Math. Appl. Med. Biol., 17:395-413, 2000.

[39] M. Shearer. Admissibility criteria for shock wave solutions of a system of conservation laws of mixed type. Proc. Roy. Soc. Edinburgh Sect. A, 93(3-4):233-244, 1982/83. MR0688788 
[40] M. Shearer. Nonuniqueness of admissible solutions of Riemann initial value problems for a system of conservation laws of mixed type. Arch. Rational Mech. Anal., 93(1):45-59, 1986. MR0822335

[41] M. Slemrod. Admissibility criteria for propagating phase boundaries in a van der Waals fluid. Arch. Rational Mech. Anal., 81(4):301-315, 1983. MR0683192

[42] M. Slemrod. Dynamic phase transitions in a van der Waals fluid. J. Differential Equations, 52(1):1-23, 1984. MR0737959

[43] M. Slemrod. Dynamics of first order phase transitions. In Phase transformations and material instabilities in solids (Madison, Wis., 1983), volume 52 of Publ. Math. Res. Center Univ. Wisconsin, pages 163-203. Academic Press, Orlando, FL, 1984. MR0802225

[44] V. L. Streeter. Fluid Mechanics. McGraw-Hill, 1971.

[45] F. M. White. Fluid Mechanics. McGraw-Hill, Boston, fourth edition, 2003.

Andrea Corli

Department of Mathematics and Computer Science

UNIVERSITY OF FERRARA

44100 FERRARA

ITALY

E-mail address: andrea.corli@unife.it

HAITAO FAN

Department of Mathematics

Georgetown University

WASHINGTON, DC 20057

USA

E-mail address: fan@math.georgetown.edu

Received August 29, 2013 